\title{
Healthy Life Style, a Strategic Way to Combat Elderly Health Problems
}

\author{
Silpa Somavarapu \\ Department of Food Technology, Vikrama Simhapuri University, Nellore, India
}

Email address:

Silpasomavarapu1981@gmail.com

\section{To cite this article:}

Silpa Somavarapu. Healthy Life Style, a Strategic Way to Combat Elderly Health Problems. Journal of Family Medicine and Health Care. Vol. 3, No. 4, 2017, pp. 75-78. doi: 10.11648/j.jfmhc.20170304.12

Received: September 10, 2017; Accepted: September 22, 2017; Published: November 30, 2017

\begin{abstract}
In India old age refers to $>60$ years. Percentage of elderly is expected to rise from $7.5 \%$ in 2010 to $11.1 \%$ in 2025 . Changes in physical, psychological, socio, economic status in old age adversely affects the individual's way of life and can lead to a variety of health consequences. Negative consequence of digitalization, urbanization and nuclear families led to unhealthy adulthood which results in serious problems related to health, nutrition and psychological problems in old age. Certain health problems in old age include hypertension, diabetes, bronchial asthma, heart diseases and kidney diseases of which nutritional problems are osteoporosis, osteomalacia, malnutrition, anemia, skin problems, cataract and psychological problems are Alzheimer, schizopherenia, different phobias, mood disorders, and personality disorders, which influence their quality of life. Several studies also showed that those who maintain healthy life styles like eating habits and regular exercise from childhood onwards will be able to control the onset and progression of diseases in elderly. Healthy diet with inclusion of high protein, fiber foods, balanced amount of vitamin, mineralized foods has a positive effect on old age. Even moderate exercise and physical activity can improve the health of people who are frail or who have diseases that accompany aging. Being physically active can also help stay strong and fit enough to keep doing the things you like to do as you get older. Taking the nutritious diet, doing good exercise and making physical activity a regular part of one's life can improve health and maintain independent, secure, happy old age. Regular health check ups and occasions to spend with family members and friends is a clear route for happy adulthood.
\end{abstract}

Keywords: Digitalization, Urbanization, Osteoporosis, Schizopherenia

\section{Introduction}

Aging is a pattern of life changes that occurs as one grows older. In India, old age refers to $>60$ years and the elderly account for $7 \%$ of the total population which by 2050 is expected to increase to nearly 2 billion - 1 in every 5 of the earth's inhabitants. This demographic transition is due to falling mortality, falling fertility, urbanisation and migration, Population ageing and rise of non communicable diseases(NCDs) [1].

\subsection{Key Issues for Older People}

Many enter old age in poor health - physical labour, poor diet/environment [2] and multiple pregnancies.

Health services are rarely "age friendly" - physically inaccessible, low levels of knowledge and negative attitudes.

\subsection{Normal Effects of Aging on the Body}

\subsubsection{Physical Changes}

Number of physical changes are seen in human beings which include changes in hair, eyesight, face, bones, joints etc.

\subsubsection{Changes in the Mind}

Typical mental changes include changes in intelligence, memory and other problems like adaptability, depression and senility.

\subsubsection{Health Issues}

Certain health problems in old age include hypertension, diabetes, bronchial asthma, cataract, heart diseases and kidney diseases.

Nutritional problems are osteoporosis, osteomalacia, 
malnutrition, anemia, skin problems, eye problems etc.

Psychological problems are Alzheimer, schizophrenia, different phobias, mood disorders, and personality disorders, which influence their quality of life.
Frailty: "The Dwindles"- It means tomeet three of five symptoms namely decreased walking speed, decreased grip strength, decreased physical activity, exhaustion and weight loss.

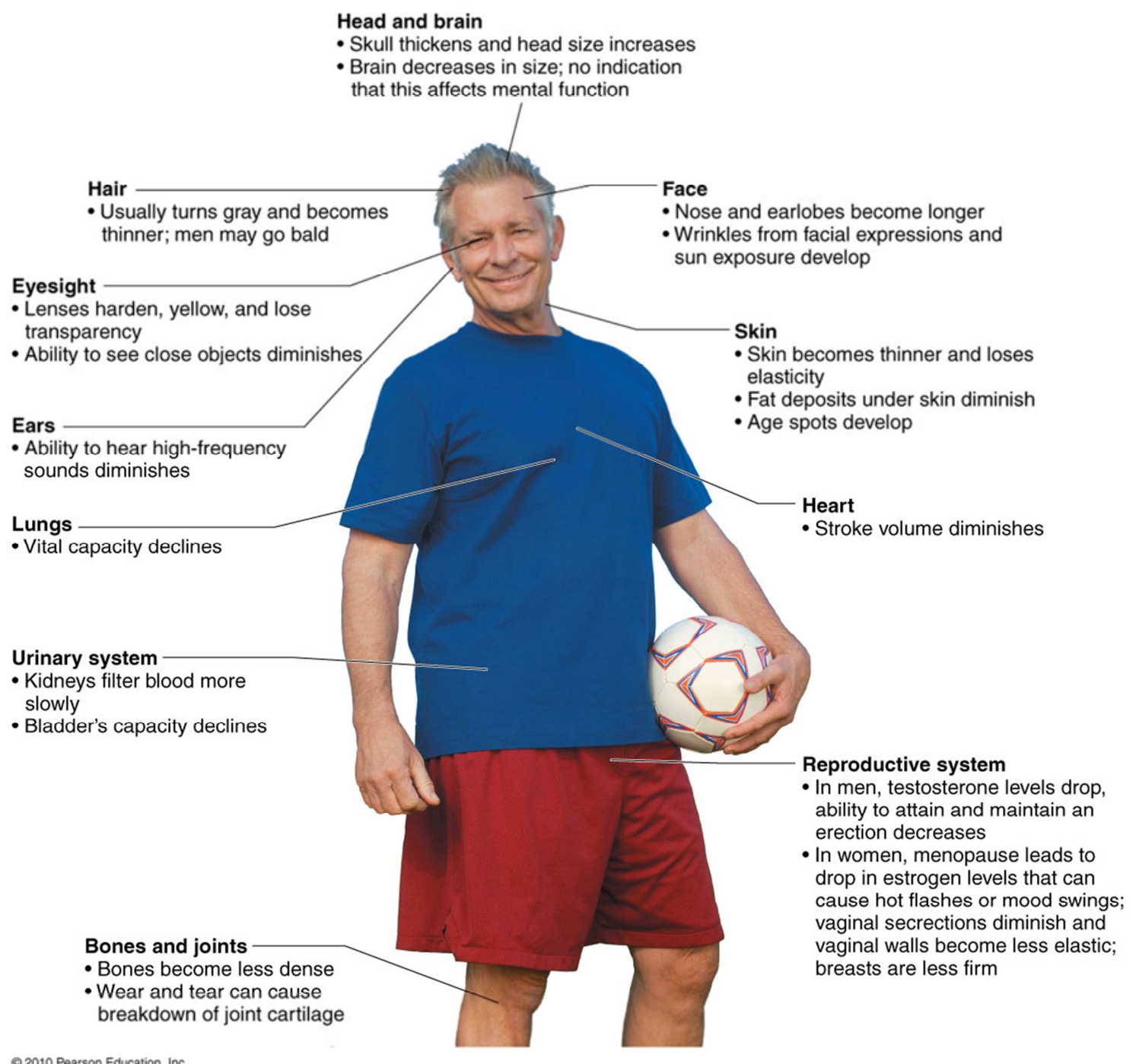

Figure 1. Typical physical changes.

\section{Methodology}

\subsection{Most Common Chronic Conditions of the Elderly and Care}

The most common chronic diseases [3] afflicting the elderly have been identified and also an efficient way of handling the condition has been elucidated

\subsubsection{Hearing Impairments}

The ability to hear well decreases with age and thus hearing impairments largely affect the elderly.

Care: Nowadays, advances in hearing aids technology may help for better hearing.

\subsubsection{Musculoskeletal Problems}

Osteoarthritisinflammation of joints due to wear and tear,
Osteoporesis (bone loss), loss of muscle mass, fractures. Care: Proper rest and exercise

\subsubsection{COPD}

COPD, or chronic obstructive pulmonary disease, is a respiratory disorder that affects the elderly's ability to breath. This leads to shortness of breath, early fatigue and low energy among other symptoms.

Care - Proper body alignment and positioning, alternate activity with periods of rest, avoid polluted air.

\subsubsection{Heart Disease}

Heart disease is one of the leading cardiovascular diseases [4] affecting the elderly.

Other related diseases include coronary artery disease or a heart attack, which are typically preceded by a poor diet, exercise regimen and hereditary factors. 
Care - Moderate exercise, according to individual's tolerance and decreased fat intake.

\subsubsection{Diabetes}

Diabetes is a result of poor insulin regulation in the body and can lead to numerous other serious health risks. Diabetes (impaired control of blood glucose), menopause, thyroid dysfunction, high blood cholesterol, are all hormonal imbalances.

Care - healthy life style, balanced diet and proper exercise.

\subsubsection{Hypertension}

Hypertension, or high blood pressure, is a condition typically defined by a resting blood pressure that is greater than $120 / 80$. It may be a primary condition in itself or a leading risk factor for several related cardiovascular diseases.

Care - food low in salt [5].

\subsection{Other Health Problems}

Arthritis: One of the most common and chronic diseases of the elderly, arthritis results in decreased joint mobility, stiffness as well as joint aches and pains.

Visual: Macular degeneration, glaucoma, cataract.

Kidneys: poor kidney function, urinary incontinence, urinary urgency, difficulty in urinating.

Cancers: prostate, colon, lung, breast, skin, bladder, ovary, brain, pancreas, only to name a few.

Bone marrow and immune system: inability to produce sufficient blood cells (anemia, myelodysplasia).

Gastrointestinal: stomach ulcers, diverticulosis (small pockets forming in the wall of colon), colon inflammation or colitis from infection or ischemic (poor blood flow) swallowing difficulty (dysphagia), constipation, bowel incontinence, hemorrhoids.

Oral and dental: gum disease, dry mouth, loss of teeth.

Infections: urinary tract infections, pneumonia, skin infections, colon infection.

Mental Disorders and Aging- Among the most common disorders dementia, depression, anxiety disorders and sleep disturbances are more common.

Parkinson's disease- Parkinson's disease is a common disease of the elderly that stems from changes in the brain which affects the nervous system. Common symptoms included tremors, shaking, difficulty in mobilizing and poor balance.

Alzheimer's disease- Alzheimer's disease is one of the common forms of dementia in the elderly. This elderly disease affects the brain which results in cognitive losses and eventually leads to complete memory loss.

\section{Results}

The study of previous literature helped to establish the fact that old age in many cases is characterized by prevalence of major psychiatric disorders. The tendency to commit suicides is also increasing due to their health problems or being neglected by their children or with an intention not to be a burden to their children and many other factors. The present scenario is to worsen by 2030 .

\section{Estimated Prevalence of Major Psychiatric Disorders by Age Group}
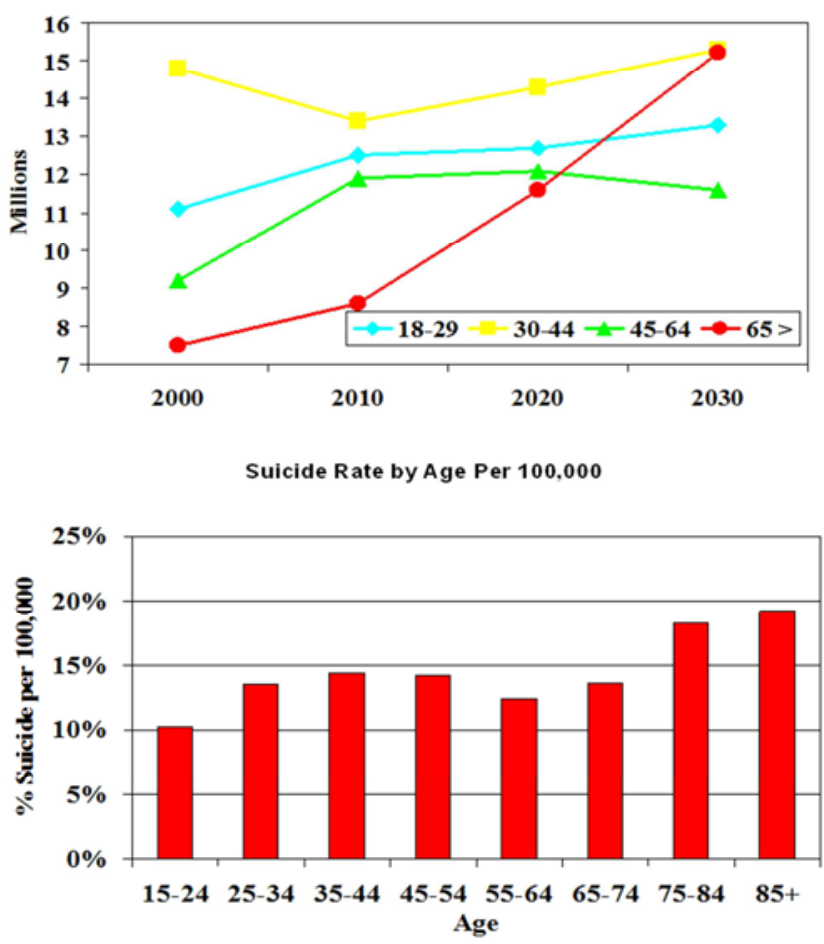

Figure 2. Estimated prevalence of major psychiatric disorders by age group and suicidal rate among elderly.

\section{Discussion}

\subsection{Healthy Life Style, a Way for Happy and Healthy Oldage}

The sure, safe and the only route for happy and healthy old age is only a good life style practice from childhood or atleast from the point where you are now. Good life style practices [6] include

\subsubsection{Exercise}

Exercise greatly benefits psychological, physical and social well being of an individual.

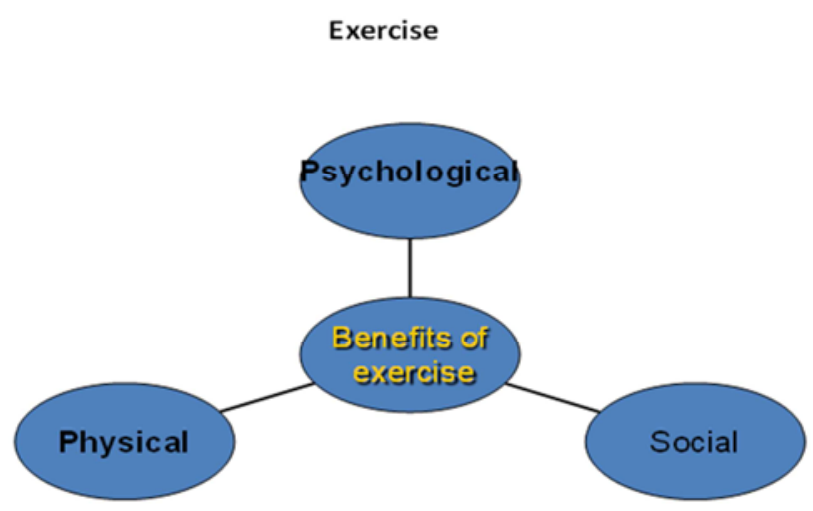

Figure 3. Benefits of exercise. 


\subsubsection{Nutrition}

A balanced diet and participation in regular exercise are paramount in maintaining a healthy life for people of all ages. Routine exercise and healthy diet in seniors can have an even more noticeable impact in their general well-being. Dietary guidelines [7] [8] for old persons include

Eat a variety of food

Maintain a healthy weight

Choose a diet low in fat, saturated and cholesterol

Choose a diet plenty of vegetables, fruits and grain products

Use sugar and salts in moderate

Drink 3-4litres of water daily.

\subsubsection{Rest \& Sleep}

Elderly need 5-7 hrs at night. Importance of Rest and sleep include

Conserve energy [9]

Provide organ respite (rest) and emerge feeling of well being.

Restore the mental alertness and neurological efficiency and relieves tension.

\subsubsection{Periodic Medical Check up}

Awareness should be increased among elderly that periodical medical check up is essential for happy and healthy old age.

Table 1. Types of health screening [10].

\begin{tabular}{ll}
\hline Health screening & Period \\
\hline Bl. P & Each Dr. visit or 3-6 months \\
Ht \& wt & Periodically as part of \\
Dental check up & physical examination \\
Fecal occult blood and sigmoidoscopy & Once / year (annually) \\
Vision including glaucoma test & Ennually) \\
Hearing & Every 2 years \\
Cholesterol level & Evaluate periodically \\
Cancer screening & Every 5 years \\
Mammography for women under 70 y & Annually \\
Digital rectal examination & $1-2$ years \\
\hline
\end{tabular}

\subsubsection{Immunizations}

Proper immunizations [11] at right time also add a lot to healthy old age.

Table 2. Schedule of immunizations.

\begin{tabular}{ll}
\hline Vaccination & Period \\
\hline Influenza (over 65y) & Annually (mid October to mid November) \\
Tetanus and diphtheria & $\begin{array}{l}\text { Every } 10 \text { years } \\
\text { Once at age } 65 \mathrm{y}, \text { revaccination for high risk } \\
\text { Pneumococcal vaccination } \\
\text { fatal pneumonia/6 y }\end{array}$ \\
\hline
\end{tabular}

\subsection{High Risk Behavior}

Such behavior should be avoided which includes multiple medications like analgesics, laxatives, antacids followed by cough products, eye wash, vitamins, and smoking and use of caffeine.

\section{Conclusion}

Proper exercise, balanced diet, rest, sleep, periodical medical checkup, proper immunizations form the right path for a happy and healthy oldage. Spiritual well-being is a state in which the positive aspects of spirituality are experienced, incorporated and lived by the individual and reflected into ones environment. The practice and incorporation of Spiritual Wellbeing into one's life influences and includes benefits for onesemotional well being, physical well being and mental well being. Other intervension sources for improving geriatric health include rehabilitation, health Care management, Online sources like GITT and nurse training programs to improving inadequate workforce of trained geriatric mental health providers.

\section{References}

[1] WHO. 2011a. Global Status Report on Non-communicable Diseases 2010. Geneva: World Health Organization.

[2] Buck Consultants. 2010. Working Well: A Global Survey of Health Promotion and Workplace Wellness Strategies Survey Report, November 2010. London: Buck Consultants.

[3] Centers for Disease Control and Prevention (CDC). Chronic Disease Overview. Atlanta, GA: CDC, 2008. Accessed at $<$ http://www.cdc.gov/NCCdphp/overview.htm> on October 31,2008 .

[4] Bock C, Diehl K, Schneider S, Diehm C, Litaker D. 2012. Behavioral counseling for cardiovascular disease prevention in primary care settings: a systematic review of practice and associated factors. Med Care Res Rev; 69(5):495-518.

[5] WASH. 2012. Welcome to World Action on Salt and Health Website.

[6] WHO (1986) Ottawa Charter for Health Promotion. World Health Organization, Geneva.

[7] Contento IR. 2011. Nutrition Education: Linking Research, Theory, and Practice ( $2^{\text {nd }}$ edition). Sudbury, MA: Jones and Bartlett Publishers.

[8] USDA and USDHHS. 2010. Dietary Guidelines for Americans, 2010 (7th edition) Washington DC: U.S. Government Printing Office.

[9] Van Cauwenberghe E, Maes L, Spittaels H, van Lenthe FJ, Brug J, Oppert JM, De Bourdeaudhuij I. 2010. Effectiveness of school-based interventions in Europe to promote healthy nutrition in children and adolescents: systematic review of published and 'grey' literature. Br J Nutr; 103(6):781-797.

[10] Van Stralen MM, Yildirim M, te Velde SJ, Brug J, van Mechelen W, Chinapaw MJ; ENERGY-consortium. 2011. What works in school-based energy balance behavior interventions and what does not? A systematic review of mediating mechanisms. Int J Obes (Lond); 35(10):1251-1265.

[11] Veerman JL, Van Beeck EF, Barendregt JJ, Mackenbach JP. 2009. By how much would limiting TV food advertising reduce childhood obesity? Eur J Public Health; 19(4):365-369. 